Wright State University

CORE Scholar

Physics Faculty Publications

Physics

6-2016

\title{
Photoexcited Carrier Trapping and Recombination at Fe Centers in $\mathrm{GaN}$
}

T. K. Uždavinys

S. Marcinkevičius

J. H. Leach

K. R. Evans

David C. Look

Wright State University - Main Campus, david.look@wright.edu

Follow this and additional works at: https://corescholar.libraries.wright.edu/physics

Part of the Physics Commons

\section{Repository Citation}

Uždavinys, T. K., Marcinkevičius, S., Leach, J. H., Evans, K. R., \& Look, D. C. (2016). Photoexcited Carrier Trapping and Recombination at Fe Centers in GaN. Journal of Applied Physics, 119, 215706.

https://corescholar.libraries.wright.edu/physics/1012

This Article is brought to you for free and open access by the Physics at CORE Scholar. It has been accepted for inclusion in Physics Faculty Publications by an authorized administrator of CORE Scholar. For more information, please contact library-corescholar@wright.edu. 


\section{A P J Journal of}

\section{Photoexcited carrier trapping and recombination at Fe centers in GaN}

T. K. Uždavinys, S. Marcinkevičius, J. H. Leach, K. R. Evans, and D. C. Look

Citation: Journal of Applied Physics 119, 215706 (2016); doi: 10.1063/1.4953219

View online: http://dx.doi.org/10.1063/1.4953219

View Table of Contents: http://scitation.aip.org/content/aip/journal/jap/119/21?ver=pdfcov

Published by the AIP Publishing

\section{Articles you may be interested in}

Effect of Fe-doping on nonlinear optical responses and carrier trapping dynamics in GaN single crystals

Appl. Phys. Lett. 107, 051901 (2015); 10.1063/1.4928125

Correlation of a generation-recombination center with a deep level trap in GaN

Appl. Phys. Lett. 106, 102101 (2015); 10.1063/1.4914393

Optical signatures of Ce related traps in $\mathrm{GaN}$

J. Appl. Phys. 113, 113504 (2013); 10.1063/1.4795263

Carrier trapping effects on photoluminescence decay time in In $\mathrm{Ga} N / \mathrm{Ga}$ N quantum wells with nanocluster structures

J. Appl. Phys. 101, 063511 (2007); 10.1063/1.2711148

Carrier recombination and diffusion in $\mathrm{GaN}$ revealed by transient luminescence under one-photon and twophoton excitations

Appl. Phys. Lett. 89, 172119 (2006); 10.1063/1.2370905

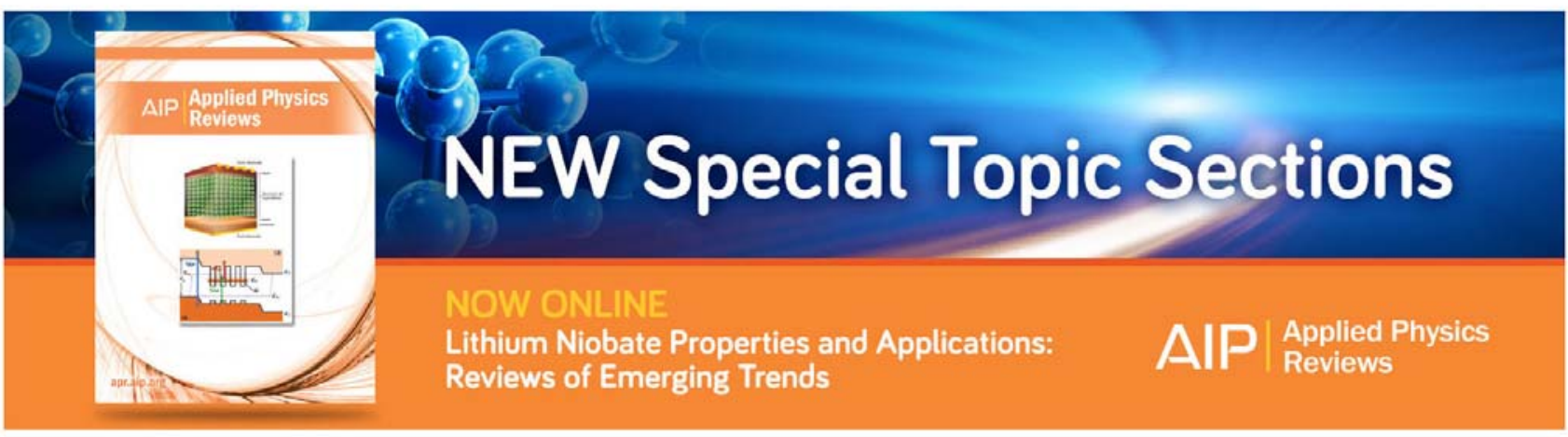




\title{
Photoexcited carrier trapping and recombination at Fe centers in GaN
}

\author{
T. K. Uždavinys, ${ }^{1}$ S. Marcinkevičius, ${ }^{1,2, a)}$ J. H. Leach, ${ }^{3}$ K. R. Evans, ${ }^{3}$ and D. C. Look ${ }^{4,5,6}$ \\ ${ }^{1}$ Department of Materials and Nano Physics, KTH Royal Institute of Technology, Electrum 229, 16440 Kista, \\ Sweden \\ ${ }^{2}$ Materials Department, University of California, Santa Barbara, California 93106, USA \\ ${ }^{3}$ Kyma Technologies Inc., 8829 Midway West Road, Raleigh, North Carolina 27617, USA \\ ${ }^{4}$ Semiconductor Research Center, Wright State University, Dayton, Ohio 45435, USA \\ ${ }^{5}$ Air Force Research Laboratory Sensors Directorate, Wright-Patterson AFB, Ohio 45433, USA \\ ${ }^{6}$ Wyle Laboratories, Inc., 2601 Mission Point Blvd., Dayton, Ohio 45431, USA
}

(Received 30 March 2016; accepted 21 May 2016; published online 7 June 2016)

\begin{abstract}
Fe doped GaN was studied by time-resolved photoluminescence (PL) spectroscopy. The shape of PL transients at different temperatures and excitation powers allowed discrimination between electron and hole capture to $\mathrm{Fe}^{3+}$ and $\mathrm{Fe}^{2+}$ centers, respectively. Analysis of the internal structure of Fe ions and intra-ion relaxation rates suggests that for high repetition rates of photoexciting laser pulses the electron and hole trapping takes place in the excited state rather than the ground state of Fe ions. Hence, the estimated electron and hole capture coefficients of $5.5 \times 10^{-8} \mathrm{~cm}^{3} / \mathrm{s}$ and $1.8 \times 10^{-8} \mathrm{~cm}^{3} / \mathrm{s}$ should be attributed to excited $\mathrm{Fe}^{3+}$ and $\mathrm{Fe}^{2+}$ states. The difference in electron capture rates determined for high (MHz) and low (Hz) (Fang et al., Appl. Phys. Lett. 107, 051901 (2015)) pulse repetition rates may be assigned to the different Fe states participating in the carrier capture. A weak temperature dependence of the electron trapping rate shows that the potential barrier for the multiphonon electron capture is small. A spectral feature observed at $\sim 420 \mathrm{~nm}$ is assigned to the radiative recombination of an electron in the ground $\mathrm{Fe}^{2+}$ state and a bound hole. Published by AIP Publishing. [http://dx.doi.org/10.1063/1.4953219]
\end{abstract}

\section{INTRODUCTION}

Fe ions in III-V and II-VI compound semiconductors act as deep acceptors and are used during the growth of semiinsulating layers for electronic and photonic devices. ${ }^{1}$ In that regard, Fe is especially important for $\mathrm{GaN}$ because the material experiences an unintentional n-type doping. Besides, Fe ions in GaN efficiently trap photoexcited carriers, producing carrier lifetimes in the ps range. ${ }^{2}$ The ultrashort carrier lifetimes, the semi-insulating properties, and the large breakdown field enable applications in the ultrafast high power optoelectronics. In addition, GaN:Fe may be interesting for spintronics because of the predicted room temperature ferromagnetism. $^{3}$

In order to reveal the full potential of $\mathrm{GaN}: \mathrm{Fe}$, properties of Fe centers, such as the electronic structure and the carrier trapping dynamics, must be determined and understood. The electronic structure of Fe ions in GaN has been studied by photoluminescence (PL), PL excitation, absorption, and electronic paramagnetic resonance and is well established. ${ }^{4,5}$ On the other hand, the reported cross-section values for the electron capture to $\mathrm{Fe}^{3+}$ vary over three orders of magnitude, between $1 \times 10^{-13} \mathrm{~cm}^{-2}$ and $4.6 \times 10^{-16} \mathrm{~cm}^{-2} \cdot 2,6,7$ Estimation of the cross section for the hole capture to $\mathrm{Fe}^{2+}$ is limited to a single study performed by time-resolved PL. ${ }^{2}$ The rich level structure of Fe ions in $\mathrm{GaN}$ formed by Stark effect, spin-orbit coupling, and Jahn-Teller effect ${ }^{4,5}$ has never been considered while discussing the carrier capture.

In this work, we study carrier trapping to Fe centers in $\mathrm{GaN}$ with the help of time-resolved PL spectroscopy.

${ }^{a)}$ Electronic mail: sm@kth.se
Excitation power dependence of PL decay and unusual transient shapes at high excitation powers allowed an unambiguous separation between the electron and hole trapping processes. Most importantly, comparison of the experimental data with previous reports and analysis of the internal structure of the $\mathrm{Fe}$ ions suggest that at high photoexciting pulse repetition rates excited rather than ground states of $\mathrm{Fe}$ ions are most relevant for the carrier trapping.

\section{EXPERIMENTAL DETAILS}

The studied samples were grown in a custom designed hydride vapor phase epitaxy (HVPE) reactor on sapphire substrates. The GaN layers were grown to a thickness of $\sim 1.5 \mathrm{~mm}$, removed from the substrates, and polished to a thickness of $\sim 0.5 \mathrm{~mm}$. Fe and impurity atom concentrations were evaluated by secondary ion mass spectrometry (SIMS) and are presented in Table I.

Time-resolved PL measurements were performed using frequency-tripled Ti:sapphire laser pulses at $269 \mathrm{~nm}$ (4.61 eV) central wavelength, a 200 fs pulse duration, and a $76 \mathrm{MHz}$ pulse repetition frequency. A spectrometer and a

TABLE I. Dopant and impurity concentrations in the studied samples.

\begin{tabular}{lcccc}
\hline \hline Sample name & {$[\mathrm{Fe}]\left(\mathrm{cm}^{-3}\right)$} & {$[\mathrm{O}]\left(\mathrm{cm}^{-3}\right)$} & {$[\mathrm{Si}]\left(\mathrm{cm}^{-3}\right)$} & {$[\mathrm{C}]\left(\mathrm{cm}^{-3}\right)$} \\
\hline $1.7 \times 10^{18}$ & $1.7 \times 10^{18}$ & $\leq 2.0 \times 10^{16}$ & $1.2 \times 10^{16}$ & $1.3 \times 10^{17}$ \\
$2.4 \times 10^{18}$ & $2.4 \times 10^{18}$ & $\leq 2.0 \times 10^{16}$ & $6.8 \times 10^{16}$ & $1.1 \times 10^{17}$ \\
$5.6 \times 10^{18}$ & $5.6 \times 10^{18}$ & $\leq 2.0 \times 10^{16}$ & $1.5 \times 10^{16}$ & $2.8 \times 10^{17}$ \\
$3.0 \times 10^{19}$ & $3.0 \times 10^{19}$ & $\leq 1.0 \times 10^{16}$ & $2.4 \times 10^{16}$ & $<1.3 \times 10^{17}$ \\
Undoped & $<1 \times 10^{15}$ & $2.0 \times 10^{16}$ & $1.5 \times 10^{16}$ & $<5 \times 10^{15}$ \\
\hline \hline
\end{tabular}


streak camera system with a time resolution of 4 ps were used for signal detection. To estimate the PL decay times below the resolution limit, a deconvolution procedure with the Gaussian system response function was applied. The sample temperature was varied between 4 and $290 \mathrm{~K}$ with the help of a closed cycle He cryostation. Average excitation powers were between 3 and $20 \mathrm{~mW}$. For a laser spot diameter of $30 \mu \mathrm{m}$ (full width at half maximum of a Gaussian profile), an absorption coefficient of $1.8 \times 10^{5} \mathrm{~cm}^{-1}$, and a reflection coefficient of 0.15 , this corresponds to photoexcited carrier densities (averaged over the laser spot diameter and the absorption length) immediately after the pulse between $8.7 \times 10^{17}$ and $5.8 \times 10^{18} \mathrm{~cm}^{-3}$. An undoped $\mathrm{GaN}$ sample grown under the same conditions was used as a reference.

\section{RESULTS}

\section{A. Time-integrated spectra}

PL spectra of the Fe doped samples were dominated by the band gap peak around $360 \mathrm{~nm}$. The spectra also have a much weaker broad PL peak around $420 \mathrm{~nm}(2.95 \mathrm{eV})$. A typical room temperature time-integrated $\mathrm{PL}$ spectrum, measured for sample $1.7 \times 10^{18}$, is presented in Fig. 1. The ratio between the integrated PL intensities of the $420 \mathrm{~nm}$ peak and band gap emission was around $2 \%-3 \%$. For sample $3.0 \times 10^{19}$, due to the weak overall PL intensity, the $420 \mathrm{~nm}$ peak could not be detected.

While the origin of the band gap PL peak is clear, transitions causing the $420 \mathrm{~nm}$ peak are not well established, even though PL at energies between 2.8 and $3.0 \mathrm{eV}$ is often observed for high-resistivity HVPE grown GaN.$^{8-10}$ Previously, the peak was assigned to transitions between a deep donor (probably a nitrogen vacancy) and the valance band, ${ }^{9}$ the conduction band and a deep acceptor (probably a carbon impurity), ${ }^{11}$ and the conduction band and a $\mathrm{H}$ and $\mathrm{C}$ complex. ${ }^{12}$ We did not observe the $420 \mathrm{~nm}$ peak in the control sample. Thus, the origin of the $420 \mathrm{~nm}$ peak in our samples is most likely related to Fe doping. Following the Fe ion energy scheme, the transition energy

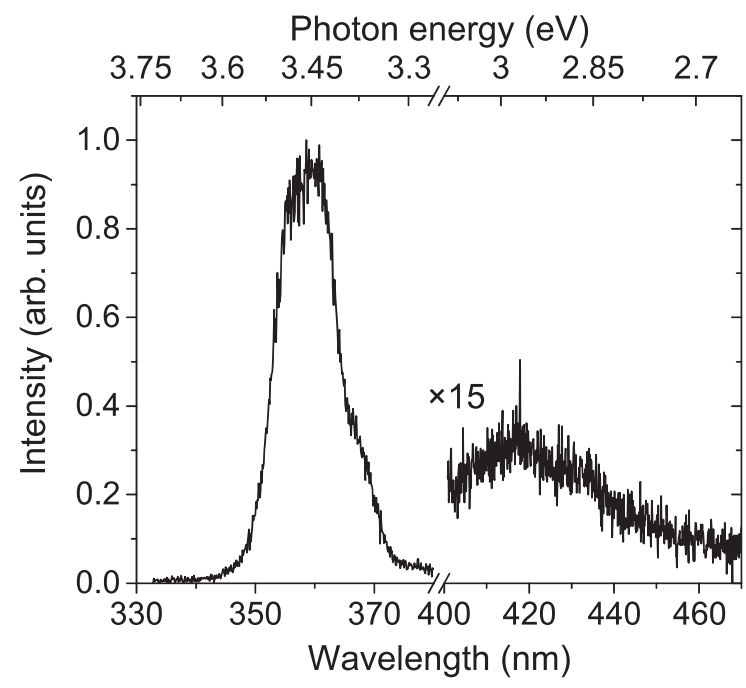

FIG. 1. Time integrated PL spectra for sample $1.7 \times 10^{18}$ at $290 \mathrm{~K}$.
$(2.95 \mathrm{eV})$ is similar to that of the absorption band in $\mathrm{Fe}^{2+} .5 \mathrm{~A}$ possible recombination mechanism for this PL peak will be discussed in Section IV.

\section{B. Photoluminescence transients at room temperature}

Spectrally integrated band gap PL transients for $13 \mathrm{~mW}$ average excitation power are shown in Fig. 2. For samples $1.7 \times 10^{18}$ to $5.6 \times 10^{18}$, the PL decay is single exponential. PL decay times, determined after deconvolution of the system response from the signal, are between 8 and 2 ps. For sample $3.0 \times 10^{19}$, the transients have a shape of the system response. Therefore, for this sample the decay times were evaluated by comparing its time-integrated PL intensities to that of other samples, for which the decay times could be measured directly. In this evaluation, the same value of the transition matrix element for all the samples was assumed. The PL decay in GaN:Fe is much faster than that in the undoped GaN sample (inset to Fig. 2). In the latter case, the PL decay is nonexponential. The initial faster decay occurs due to the carrier diffusion away from the surface and deeper into the sample, ${ }^{13}$ while the slower decay at longer times may be ascribed to the nonradiative recombination through native defects. ${ }^{14}$ A similar ultrafast PL decay as in $\mathrm{GaN}: \mathrm{Fe}$ was previously observed for $\mathrm{Fe}$ doped $\mathrm{InP}$ and InGaP. ${ }^{15,16}$ Short carrier lifetimes in $\mathrm{GaN}$ :Fe were also detected in pump-probe experiments. ${ }^{6}$ These studies have shown that $\mathrm{Fe}$ ions act as efficient nonradiative recombination centers.

PL transients for the $420 \mathrm{~nm}$ peak (Fig. 3) in the initial part of the decay can be described by a single exponential as well. The decay times are 69,57 , and 24 ps for samples $1.7 \times 10^{18}, 2.4 \times 10^{18}$, and $5.6 \times 10^{18}$, respectively. These times are too short to be assigned to the radiative recombination; on the other hand, they are an order of magnitude longer than the ultrafast carrier trapping times measured at the band gap (Fig. 2). For the $420 \mathrm{~nm}$ peak, no significant PL

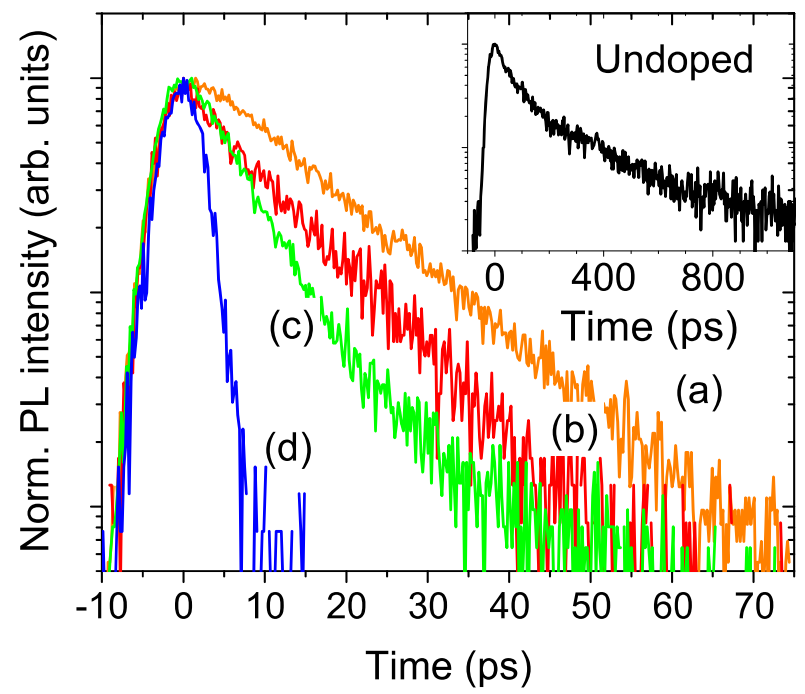

FIG. 2. $290 \mathrm{~K}$ normalized band gap PL transients for samples with different Fe concentrations at $13 \mathrm{~mW}$ average excitation power; sample $1.7 \times 10^{18}$ (a), $2.4 \times 10^{18}$ (b), $5.6 \times 10^{18}$ (c), and $3.0 \times 10^{19}(\mathrm{~d})$. The inset (log scale) shows PL dynamics for the undoped sample. 


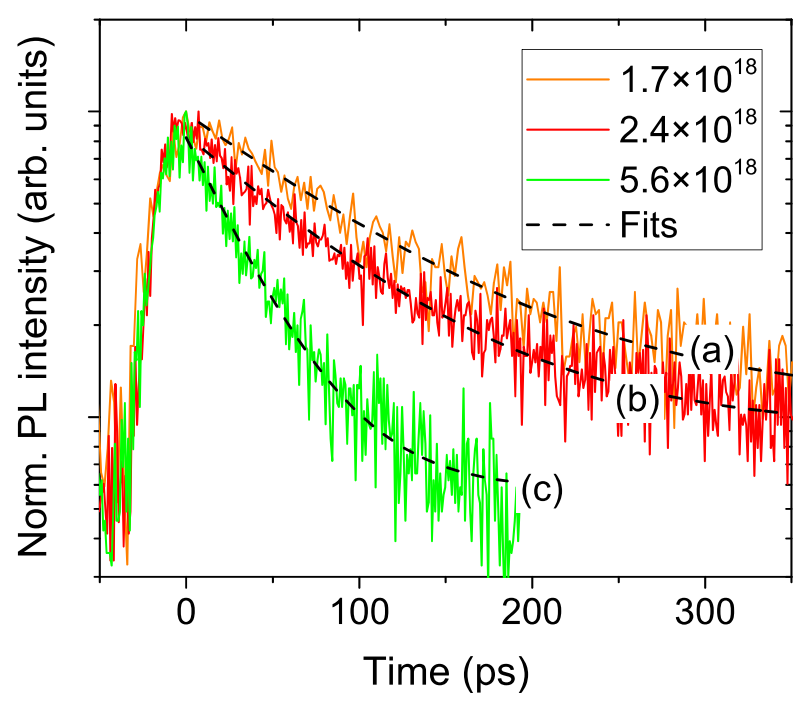

FIG. 3. Normalized $420 \mathrm{~nm}$ peak PL transients for samples $1.7 \times 10^{18}$ (a), $2.4 \times 10^{18}(\mathrm{~b})$, and $5.6 \times 10^{18}(\mathrm{c})$ measured at $10 \mathrm{~mW}$ average excitation power at $290 \mathrm{~K}$.

decay time dependence on the excitation power in $1-10 \mathrm{~mW}$ range was observed.

\section{Carrier trapping dynamics}

After a laser pulse, photoexcited carriers are captured by Fe traps and charge transfer processes, in a simplified form described as $\mathrm{Fe}^{3+}+$ electron $\rightarrow \mathrm{Fe}^{2+}$ and $\mathrm{Fe}^{2+}+$ hole $\rightarrow$ $\mathrm{Fe}^{3+}$, take place. Carrier trapping and recombination through Fe ions affect the PL decay time $\tau_{P L}$

$$
\begin{aligned}
\frac{1}{\tau_{P L}} & =\frac{1}{\tau_{e}}+\frac{1}{\tau_{h}}=c_{e}\left[F e^{3+}\right]+c_{h}\left[F e^{2+}\right] \\
& =\bar{v}_{e} \sigma_{e}\left[F e^{3+}\right]+\bar{v}_{h} \sigma_{h}\left[F e^{2+}\right] .
\end{aligned}
$$

Here, $\tau_{e}$ and $\tau_{h}$ are electron and hole capture times, $c_{e}$ and $c_{h}$ are corresponding capture coefficients, $\bar{v}_{e}$ and $\bar{v}_{h}$ are average thermal velocities, and $\sigma_{e}$ and $\sigma_{h}$ are capture cross-sections. As pointed out in Ref. 17, the capture coefficients are more fundamental quantities because in dynamical experiments the thermal velocities are not constant. Further on, $\left[F e^{3+}\right]$ and $\left[\mathrm{Fe}^{2+}\right]$ are neutral and ionized $\mathrm{Fe}$ trap concentrations, related to the total $\mathrm{Fe}$ dopant concentration via $[\mathrm{Fe}]$ $=\left[F e^{3+}\right]+\left[F e^{2+}\right]$. However, the cross-sections have a more intuitive interpretation and are provided in most of the relevant publications. In Eq. (1), only carrier trapping to $\mathrm{Fe}-$ related centers is considered, which is justified by the slow PL decay for the reference undoped sample. Here, we assume that recombination through other impurities, defects, and surface states do not change significantly with the iron doping.

Since the concentration of unintentional donors, such as $\mathrm{Si}$ and $\mathrm{O}$, is lower than the concentration of $\mathrm{C}$ acceptors with the energy level closer to the valence band than $\mathrm{Fe}^{3+5,12}$ prior to the photoexcitation majority of $\mathrm{Fe}$ ions are in the $\mathrm{Fe}^{3+}$ state, i.e., $\left[\mathrm{Fe}^{3+}\right] \gg\left[\mathrm{Fe}^{2+}\right]$. To get a qualitative picture on the photoexcited carrier capture processes, let us examine two extreme cases:
For low excitation $(\Delta n<[F e]$, where $\Delta n$ is the photoexcited electron concentration), the PL dynamics are primarily determined by the electron trapping to $\mathrm{Fe}^{3+}$, the first term of Eq. (1). Under such conditions, the electron capture rate is proportional to the total $\mathrm{Fe}$ concentration, and the capture coefficient can be evaluated in a straightforward way. Fig. 4 shows electron capture rates (inverse PL decay times) estimated from the single exponential decay at low and high temperatures for low excitation power as a function of $\mathrm{Fe}$ concentration. The capture rate increases proportionally with the Fe dopant concentration, which allows evaluating the electron capture coefficient $c_{e}=7.5 \pm 2.0 \times 10^{-8} \mathrm{~cm}^{3} / \mathrm{s}$. Surprisingly, there is little difference between the capture rates for different temperatures. This observation is discussed in Section III E.

Under high excitation conditions $(\Delta n>[F e])$, and if the hole capture coefficient to $\mathrm{Fe}^{2+}$ is not much larger than the coefficient of electron capture to $\mathrm{Fe}^{3+}$, majority of $\mathrm{Fe}^{3+}$ traps get filled with electrons during or shortly after the excitation leading to $\left[\mathrm{Fe}^{2+}\right]>\left[\mathrm{Fe}^{3+}\right]$. In this case, the initial decay would be dominated by the second term of Eq. (1). After some time, $\mathrm{Fe}^{2+}$ concentration would decrease due to the hole capture, and eventually the system would reach a dynamical equilibrium.

To illustrate these two situations, we provide examples of excitation power dependent PL transients for sample $5.6 \times 10^{18}$ at $4 \mathrm{~K}($ Fig. $5(\mathrm{a}))$ and sample $1.7 \times 10^{18}$ at $290 \mathrm{~K}$ (Fig. 5(b)). When $\Delta n<[F e]$, the PL decay is single exponential. With an increased power (intermediate conditions), the decay time increases, as the hole capture becomes nonnegligible. For high excitation powers, an unusual double exponential decay shape develops with the initial decay 2 to 3 times slower compared to the subsequent faster component. Qualitatively, one can understand the high-excitation situation as follows. Due to the fast electron trapping to $\mathrm{Fe}^{3+}$, electrons excited at high densities quickly transform most of $\mathrm{Fe}^{3+}$ ions into $\mathrm{Fe}^{2+}$. Thus, the slower PL decay should be mainly determined by the hole trapping. The following faster decay is comparable to the decay at intermediate powers and is slower than that for the low excitation. In this time range, $\left[\mathrm{Fe}^{3+}\right]$ and $\left[\mathrm{Fe}^{2+}\right]$ are comparable, and PL transients are

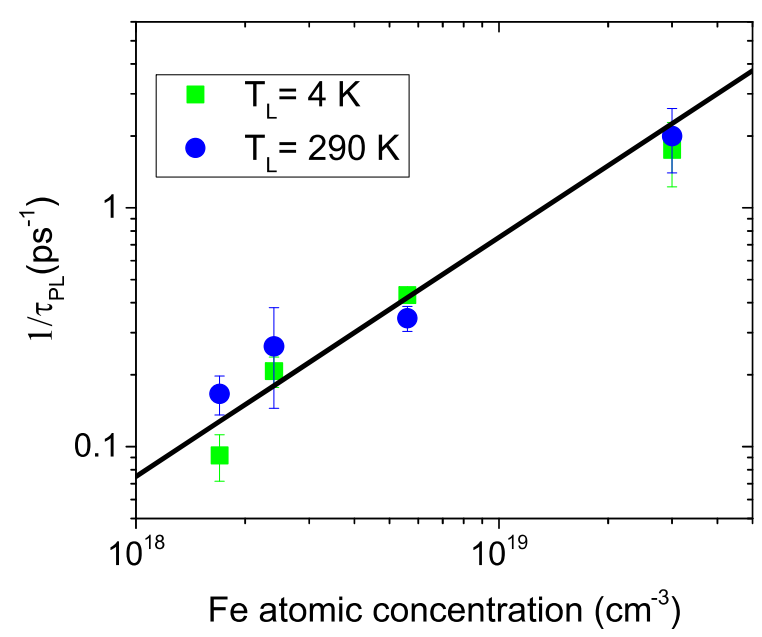

FIG. 4. Capture rate dependence on the Fe concentration for $3 \mathrm{~mW}$ average excitation power at $4 \mathrm{~K}$ and $290 \mathrm{~K}$. 

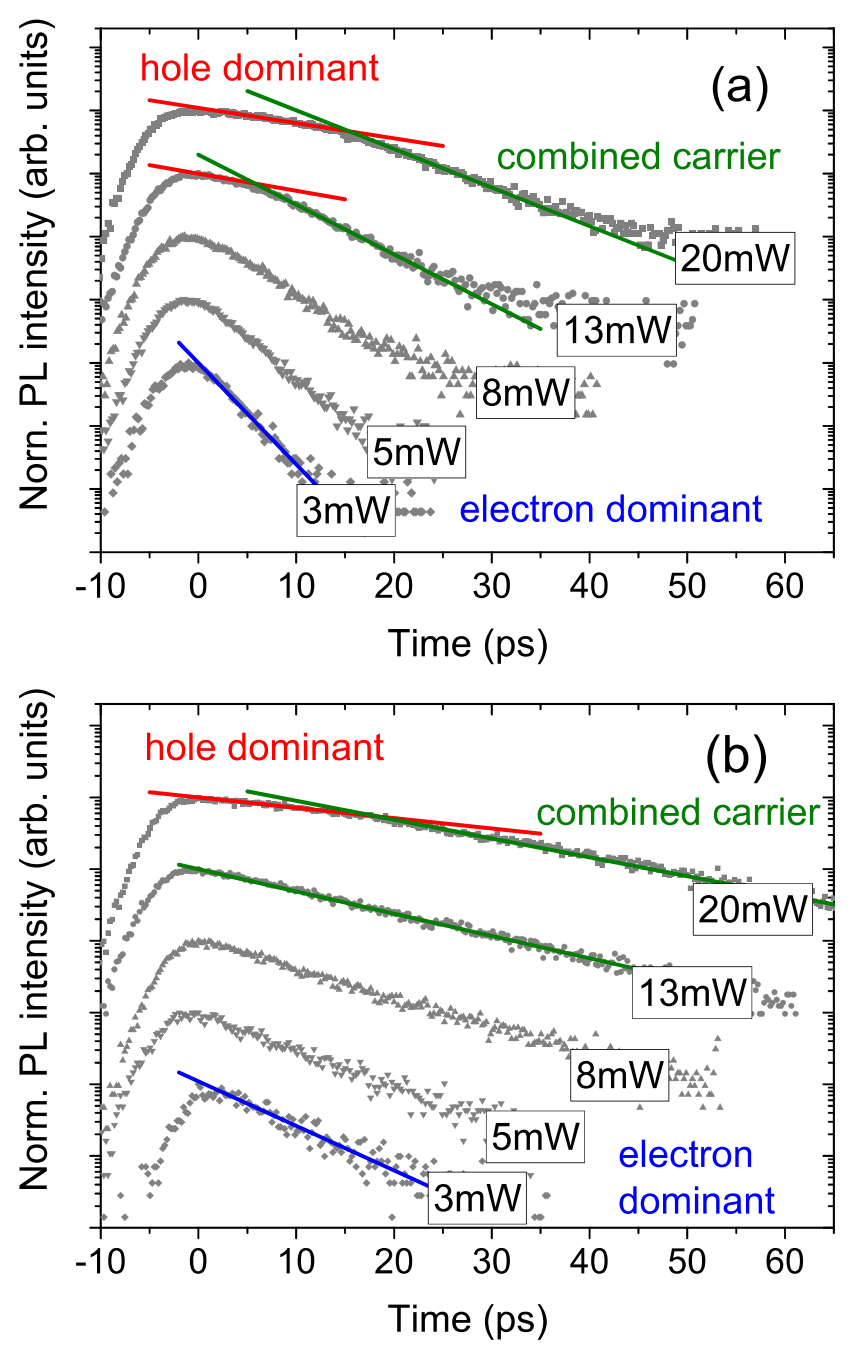

FIG. 5. Excitation dependent PL transients: sample $5.6 \times 10^{18}$ at $4 \mathrm{~K}$ (a) and sample $1.7 \times 10^{18}$ at $290 \mathrm{~K}(\mathrm{~b})$.

determined by both terms of Eq. (1). In Fig. 5, this regime is defined as combined carrier trapping.

At $4 \mathrm{~K}, \mathrm{Fe}^{3+}$ saturation was observed in all the samples but the one with the highest doping $\left(3 \times 10^{19}\right)$. At room temperature, this effect was observed only for the sample with the lowest $\mathrm{Fe}$ concentration $\left(1.7 \times 10^{18}\right)$. This difference may be assigned to the thermal activation of other nonradiative recombination channels, not considered in Eq. (1). These could be native defects, such as Ga vacancies (dominant in undoped $\mathrm{GaN}),{ }^{14,18} \mathrm{C}$ impurities, ${ }^{12}$ and $\mathrm{N}$ vacancies (the concentration of which is expected to increase with the Fe doping). ${ }^{10}$ More details on the interplay between $\mathrm{Fe}^{3+}$ and $\mathrm{Fe}^{2+}$ centers will be provided below. However, already from this simple qualitative evaluation one can conclude that the hole trapping rate is slower than the electron trapping rate, otherwise the double exponential shape with the initial slow component could not be observed.

\section{Numerical modeling}

To support these qualitative considerations and retrieve the hole trapping coefficient, simple numerical simulations were performed. In general, at low to moderate photoexcited carrier concentrations, carrier dynamics and PL transients are affected by radiative recombination, carrier trapping, recombination at deep centers and surface states, PL reabsorption, and carrier diffusion. However, the ultrafast carrier trapping to $\mathrm{Fe}$ centers resulting in ultrashort carrier lifetimes allows discarding the majority of these effects, which considerably simplifies the description of the PL transients. As mentioned (Fig. 2), comparison with the undoped sample suggests that radiative recombination and carrier trapping to impurities, native defects and surface states are much slower than their trapping to Fe centers. This assumption is supported by the following arguments. First, the SIMS data show that $\mathrm{Fe}$ doping does not introduce impurities, such as $\mathrm{O}, \mathrm{C}$, and Si (Table I). Then, Fe doping has been found to decrease the concentration of Ga vacancies. ${ }^{10}$ Finally, we do not anticipate that Fe doping considerably modifies the surface state concentration. At least in GaAs:Fe, where Fe is not the dominant carrier trap, doping with iron has no major influence on the carrier trapping rates. ${ }^{19}$ Concerning the lateral carrier diffusion in the sample plane, its length is negligible in comparison to the excitation spot size. The PL reabsorption in samples with short carrier lifetimes can also be neglected. ${ }^{13}$ Thus, besides the Fe-related trapping, the only effect that might influence the PL transients by changing the carrier concentration is the carrier diffusion toward the bulk of the sample. However, this effect is only important when the photoexcited carrier concentration is similar to that of Fe dopants. For high Fe doping and low photoexcited carrier concentration, $\Delta n \ll[F e]$, decrease of the carrier concentration due to diffusion does not affect the trapping rate. For high excitation, diffusion can still be neglected at early times, during which it does not affect the carrier concentration substantially and the relation $\Delta n>[\mathrm{Fe}]$ is maintained for most of the carriers. For example, during the first 10 ps after the excitation, the average carrier concentration within the first absorption length decreases by only $\sim 20 \%$ (in the estimation, the ambipolar diffusion coefficient of $1.6 \mathrm{~cm}^{2} / \mathrm{s}$ (Ref. 20) was used).

Neglecting the influence of carrier diffusion on the PL transients, the latter can be described by

$$
I(t) \propto \Delta n(t) \Delta p(t)
$$

The non-equilibrium carrier concentrations $\Delta n$ and $\Delta p$ can be found by describing the system with the rate equations that take into account the dynamic relation between $\left[F e^{3+}\right]$ and $\left[F e^{2+}\right]$

$$
\begin{gathered}
d \Delta n / d t=-c_{e}\left[F e^{3+}\right](t) \Delta n(t), \\
d \Delta p / d t=-c_{h}\left[F e^{2+}\right](t) \Delta p(t), \\
d\left[F e^{3+}\right] / d t=-c_{e}\left[F e^{3+}\right](t) \Delta n(t)+c_{h}\left[F e^{2+}\right](t) \Delta p(t), \\
d\left[F e^{2+}\right] / d t=c_{e}\left[F e^{3+}\right](t) \Delta n(t)-c_{h}\left[F e^{2+}\right](t) \Delta p(t) .
\end{gathered}
$$

The initial conditions are: $\Delta n=\Delta p,\left[F e^{3+}\right]=[F e]$, and $\left[F e^{2+}\right]=0$.

Let us first consider the situation $\Delta n<[\mathrm{Fe}]$. Using the electron capture coefficient determined from the linear dependence of the PL decay rate, PL transients were calculated 
neglecting the variation of the hole concentration and the process of the trap state transfer from $\mathrm{Fe}^{2+}$ to $\mathrm{Fe}^{3+}$. To match the experimental conditions, the calculated transients were convoluted with the Gaussian system response function. The simulation results for sample $5.6 \times 10^{18}$ are shown in Fig. 6(a). The calculated transients match the experimental results up to $8 \mathrm{~mW}$ excitation, after which photoexcited electron concentration exceeds that of Fe dopants. For high excitation powers and at longer times after the excitation, the condition $\left[\mathrm{Fe}^{2+}\right]>\left[\mathrm{Fe}^{3+}\right]$ holds, and $\mathrm{Fe}^{3+}$ can no longer be considered the dominant trap (Fig. 6(b)). Neglecting the transition $\mathrm{Fe}^{2+}+$ hole $\rightarrow \mathrm{Fe}^{3+}$ affects the transients and induces a discrepancy between the experimental and the calculated PL intensity values (Fig. 6(a)).

To investigate the hole trapping, simulations of PL transients for high excitation powers were made. Results for samples $1.7 \times 10^{18}$ and $2.4 \times 10^{18}$ at $20 \mathrm{~mW}$ average power are shown in Fig. 7(a). The simulations have allowed refining the value of the electron capture coefficient that was estimated from Fig. 4. The coefficient obtained from the simulations is $5.5 \times 10^{-8} \mathrm{~cm}^{3} / \mathrm{s}$, which is within the error margin of the roughly estimated value. The room temperature electron capture cross section, adjusted for the hot carrier effect (see below), is $2.4 \times 10^{-15} \mathrm{~cm}^{2}$.
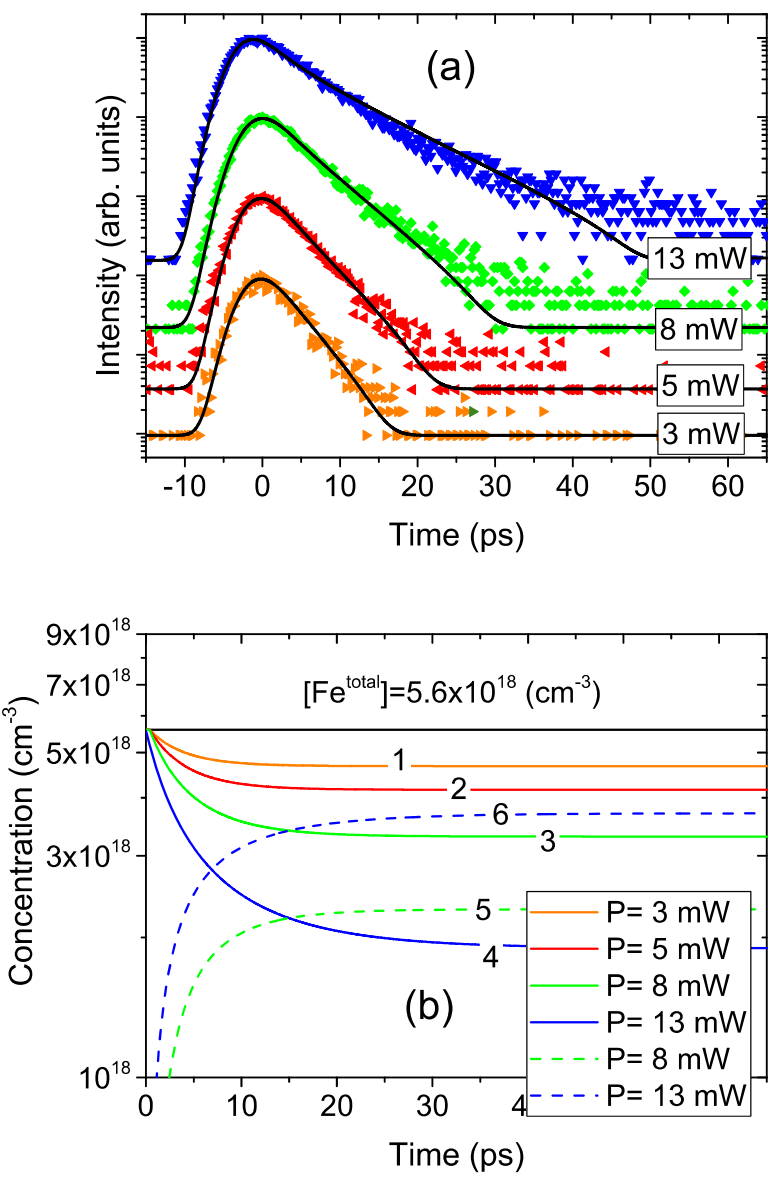

FIG. 6. Experimental (points) and calculated (curves) PL transients for sample $5.6 \times 10^{18}$ at different average excitation powers (a). Part (b) shows calculated $\mathrm{Fe}^{3+}$ and $\mathrm{Fe}^{2+}$ concentrations (solid and dashed lines, respectively) neglecting the transition $\mathrm{Fe}^{2+}+$ hole $\rightarrow \mathrm{Fe}^{3+}$. Average excitation powers in (b): $3 \mathrm{~mW}$ (curve 1), $5 \mathrm{~mW}$ (2), $8 \mathrm{~mW}$ (3 and 5), and $13 \mathrm{~mW}$ (4 and 6).
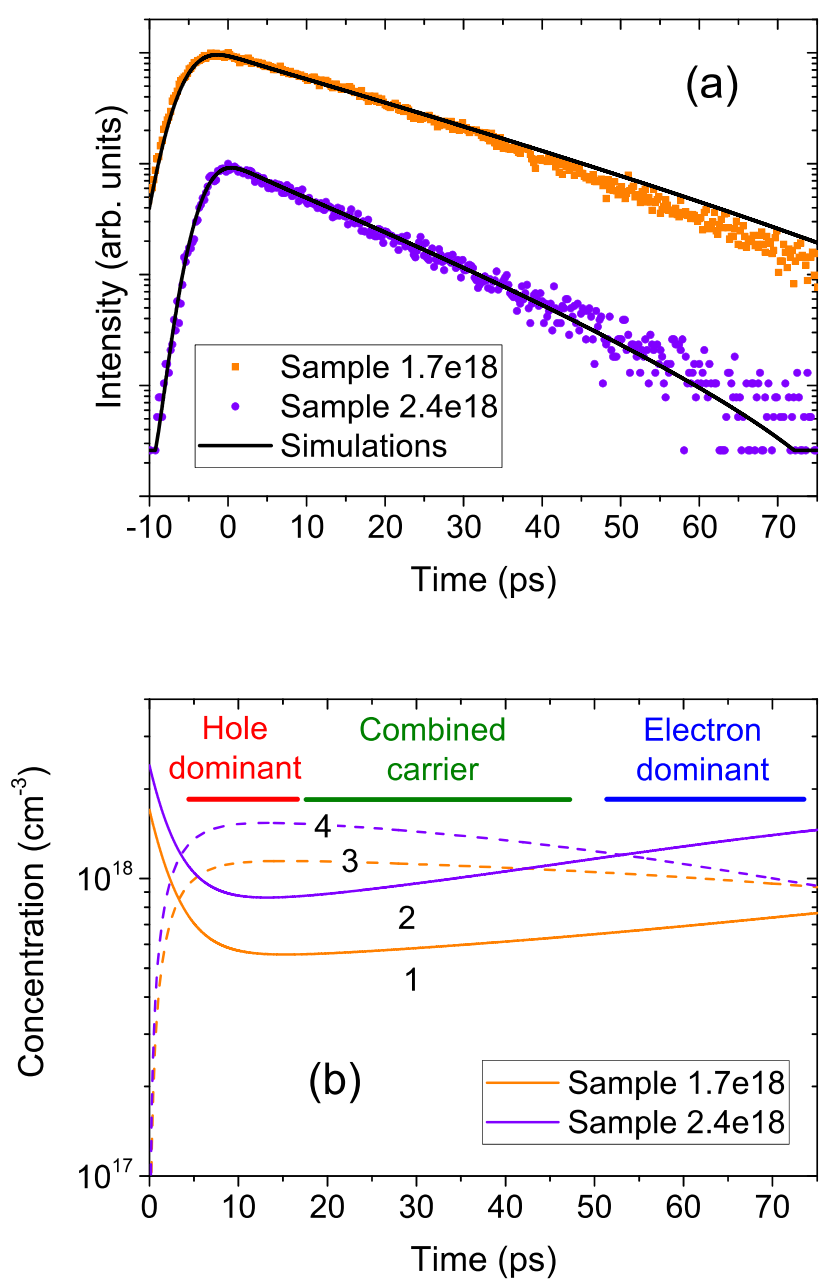

FIG. 7. Experimental (points) and calculated (lines) $290 \mathrm{~K}$ PL transients for samples $1.7 \times 10^{18}$ and $2.4 \times 10^{18}$ at $20 \mathrm{~mW}$ average power (a). Part (b) shows temporal dependence of $\left[\mathrm{Fe}^{3+}\right]$ (solid lines 1 and 2) and $\left[\mathrm{Fe}^{2+}\right]$ (dashed lines 3 and 4 ) for samples $1.7 \times 10^{18}$ (lines 1 and 3 ) and $2.4 \times 10^{18}$ (lines 3 and 4).

The hole capture coefficients were evaluated from simulations of PL transients at early times after the excitation. The best agreement between the experimental and the calculated results was obtained for the hole capture coefficient of $1.8 \times 10^{-8} \mathrm{~cm}^{3} / \mathrm{s}$. Estimation of the hole capture crosssection is complicated by the uncertainty in the hole effective mass. ${ }^{21}$ Using the average value of $1.58 m_{0}$ (Ref. 21) and the carrier temperature of $330 \mathrm{~K}$, the calculated hole capture cross-section is $2.0 \times 10^{-15} \mathrm{~cm}^{-2}$, which can be compared with the previously ${ }^{2}$ determined upper limit of $2.1 \times 10^{-15} \mathrm{~cm}^{-2}$ (recalculated for the hole mass of $1.58 m_{0}$ ).

Figure 7(b) shows time dependence of $\mathrm{Fe}^{3+}$ and $\mathrm{Fe}^{2+}$ trap concentrations. For both samples, $\mathrm{Fe}^{2+}$ becomes the dominant trap of nonequilibrium carriers within a few ps. For sample $1.7 \times 10^{18}$, the condition $\left[\mathrm{Fe}^{2+}\right]>\left[\mathrm{Fe}^{3+}\right]$ holds until about $85 \mathrm{ps}$ after the excitation. This supports the assumption made in Section III C that the more oblique slope of the PL transients reflects the hole capture. For sample $2.4 \times 10^{18}$, the time interval over which $\left[\mathrm{Fe}^{2+}\right]>\left[\mathrm{Fe}^{3+}\right]$ is shorter because of the higher $\mathrm{Fe}$ concentration leading to a faster recovery of the $\mathrm{Fe}^{3+}$ traps. 
Despite the crude simplification of the model by ignoring the spatial nonuniformity of the carrier concentration and its change due to diffusion, the calculated and experimental curves of PL dynamics match well. Primarily, this occurs because of the relatively similar electron and hole capture coefficients, which assure that there is not much difference between electron capture to $\mathrm{Fe}^{3+}$ or hole capture to $\mathrm{Fe}^{2+}$.

\section{E. Temperature dependence}

Optical measurements of carrier trapping to deep centers are almost exclusively performed at room temperature. However, measurements at low temperatures and the temperature dependence of the carrier capture are interesting for several reasons. At low temperatures, the phonon occupation number is low; hence, processes with the phonon absorption, like the thermionic emission, are eliminated. Besides, the relevance of the slow nonradiative recombination channels, mentioned above, is reduced.

PL transients, determined by the carrier capture to the $\mathrm{Fe}$ centers, show little temperature dependence (Fig. 8(a)). PL decay times, with the increase of the lattice temperature $T_{L}$ from $4 \mathrm{~K}$ to $290 \mathrm{~K}$, decrease by a factor of 2 for $1.7 \times 10^{17}$ sample, show an even smaller decrease for $2.4 \times 10^{18}$ sample and remain temperature independent for $5.6 \times 10^{18}$ sample. When dealing with the ultrafast carrier trapping, one should bear in mind that carriers may be trapped prior to their relaxation in the bands, i.e., being hot. Hot carriers more easily overcome potential barriers; thus, the carrier temperature should also be taken into account when estimating the capture cross-sections. The cross-sections, estimated using Eq. (1), are affected by the carrier temperature $T_{C}$ via the average thermal velocity $\bar{v}=\sqrt{8 k T_{C} /\left(\pi m^{*}\right)}$, where $m^{*}$ is an effective mass.

For carriers with a Boltzmann distribution, the carrier temperature $T_{C}$ can be estimated from the high-energy slope of a PL spectrum. For photon energies $h \nu$ exceeding the band gap $E_{g}, I(h \nu) \propto \exp \left(-\left(h \nu-E_{g}\right) /\left(k T_{C}\right)\right){ }^{22}$ In this work, because of the very short carrier lifetimes, temporal evolution of the carrier temperature was not considered and average carrier temperatures were estimated from time-integrated

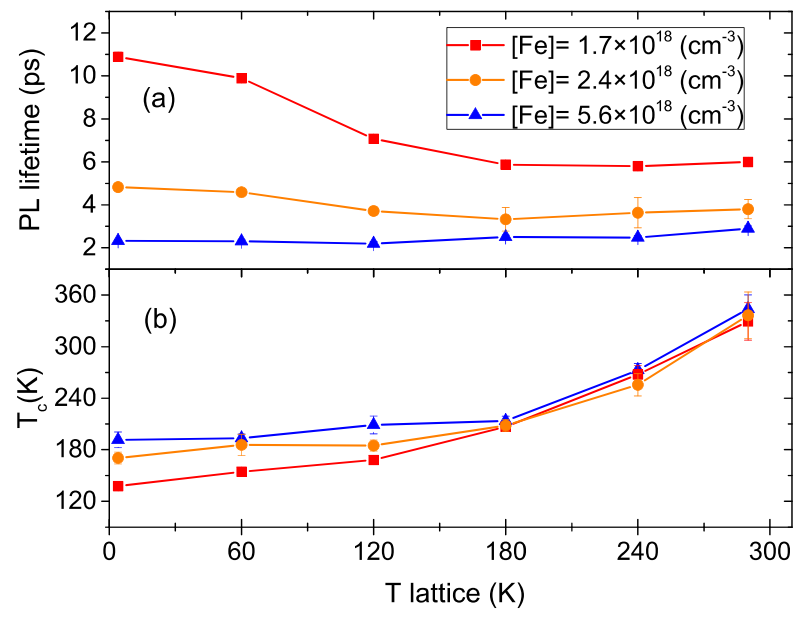

FIG. 8. PL decay times (a) and carrier temperatures (b) at $3 \mathrm{~mW}$ average excitation power for samples $1.7 \times 10^{18}, 2.4 \times 10^{18}$ and $5.6 \times 10^{18}$. spectra. The carrier temperatures at low excitation power are shown in Fig. 8(b). The average $T_{C}$ is larger than $T_{L}$ for all samples and all lattice temperatures. From the three samples shown in Fig. 8, the smallest variation of the carrier temperature with $T_{L}$ is for sample $5.6 \times 10^{18}$ with the shortest carrier lifetime. The larger $T_{C}$ variation for other samples occurs because of the longer time the carriers spend in the bands, during which they cool down. $T_{C}$ for sample $3.0 \times 10^{19}$ (not shown) was almost $100 \mathrm{~K}$ higher than $T_{L}$ because of the subps trapping.

Carrier capture to deep centers, such as $\mathrm{Fe}$ in $\mathrm{GaN}$, takes place via multiphonon absorption. ${ }^{23}$ Recent calculations of carrier capture coefficients for different defects in $\mathrm{GaN}$ and $\mathrm{ZnO}$ showed that the temperature dependence of the trapping coefficient is strongly affected by the configuration diagram of the particular defect. ${ }^{17}$ For instance, hole capture coefficients of $\mathrm{Li}_{\mathrm{Zn}}$ impurity in $\mathrm{ZnO}$ or of $\left(\mathrm{Zn}_{\mathrm{Ga}}-\mathrm{V}_{\mathrm{N}}\right)$ complex in $\mathrm{GaN}$ have a weak temperature dependence because of very small energy barriers for the hole capture. Our nearly temperature independent electron capture rates to $\mathrm{Fe}^{3+}$ suggests that this process also has a small barrier, which can be easily overcome by hot electrons.

\section{DISCUSSION}

The room temperature electron capture coefficient determined in our experiments and simulations is $5.5 \times 10^{-8} \mathrm{~cm}^{3} / \mathrm{s}$. This is close to the value of $4.6 \times 10^{-8} \mathrm{~cm}^{3} / \mathrm{s}$ obtained in similar experiments on the films grown by low-pressure metal-organic vapor phase epitaxy. ${ }^{2}$ Possible Fe clustering in the latter case might account for the difference. However, compared to recent time-resolved transmission and photo-Hall data, the difference in electron capture coefficients is much larger. The value estimated from differential transmission decay times in low pulse repetition rate $(10 \mathrm{~Hz})$ pump-probe measurements on HVPE grown bulk crystals is about $1 \times 10^{-8}$ $\mathrm{cm}^{3} / \mathrm{s}^{6}$ The coefficient evaluated from continuous wave (cw) photo-Hall experiments at very low carrier densities $\left(\sim 10^{8} \mathrm{~cm}^{-3}\right)$ on samples similar to ours is even smaller, $2.2 \times 10^{-9} \mathrm{~cm}^{3} / \mathrm{s}^{24}$ To understand why different experiments produce different results, one should consider the internal $3 d$ level structure of the Fe centers.

In equilibrium conditions, the ratio between $\mathrm{Fe}^{3+}$ and $\mathrm{Fe}^{2+}$ concentrations is determined by unintentional donors and acceptors. Besides, $\mathrm{Fe}^{3+}$ and $\mathrm{Fe}^{2+}$ centers are in their ground states, since the intra-center excitation energies ${ }^{5}$ are much larger than the thermal energy at room temperature. After electron-hole pairs are excited by a laser pulse into the bands, carrier capture by Fe traps and charge transfer processes take place. In a simplified form, the latter can be described as $\mathrm{Fe}^{3+}+$ electron $\rightarrow \mathrm{Fe}^{2+}$ and $\mathrm{Fe}^{2+}+$ hole $\rightarrow$ $\mathrm{Fe}^{3+}$. However, because of the $d$ level structure and the inter-center relaxation, these processes are subtler.

It has been reported that $\mathrm{Fe}^{3+}$ in $\mathrm{GaN}$ has four states in the band gap, and $\mathrm{Fe}^{2+}$ has two. ${ }^{5}$ The ground state of $\mathrm{Fe}^{3+}$ is ${ }^{6} \mathrm{~A}_{1}$ with the spin $\mathrm{S}=5 / 2$, and the first excited state is ${ }^{4} \mathrm{~T}_{1}$ with $S=3 / 2$ (Fig. 9). Since the relaxation ${ }^{4} T_{1} \rightarrow{ }^{6} A_{1}$ involves a spin-flip, it is slow, in the ms range. ${ }^{5}$ The relaxation times from higher excited states ${ }^{4} \mathrm{~T}_{2}$ and ${ }^{4} \mathrm{E}$ states to the 


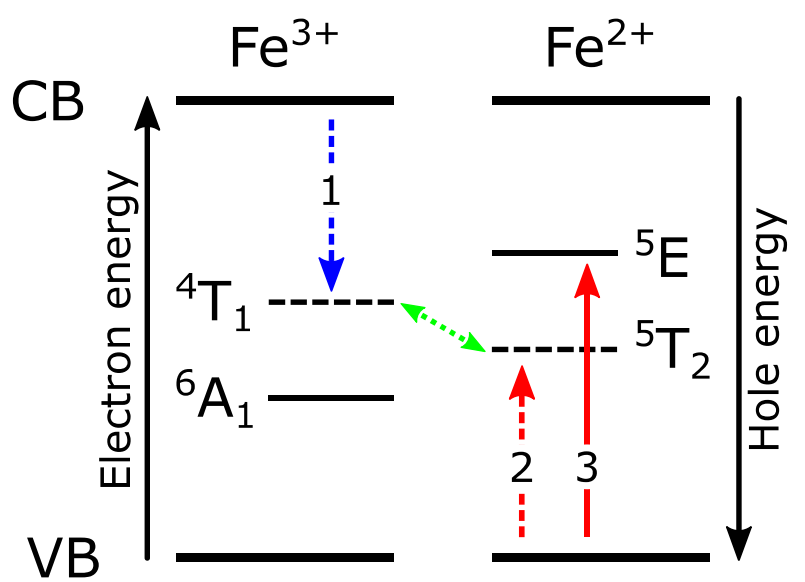

FIG. 9. Recombination model for the high pulse repetition rate timeresolved PL experiment. An electron, excited over the GaN band gap, is captured to the excited state of $\mathrm{Fe}^{3+}$ (process 1) transforming it into the excited state of $\mathrm{Fe}^{2+}$ (green dotted line), from where it recombines with a hole in the valence band (process 2) returning the ion to the excited state of $\mathrm{Fe}^{3+}$. The radiative recombination from ground state of $\mathrm{Fe}^{2+}$ is indicated as process 3. Higher $\mathrm{Fe}^{3+}$ excited states are not shown. Level positions with respect to the bands and interlevel energies are not in scale.

ground state ${ }^{6} \mathrm{~A}_{1}$ are not known; however, considering that these states are also characterized by $\mathrm{S}=3 / 2,{ }^{25}$ one could expect that the electron relaxation from the higher to the first excited state is considerably faster than the transition ${ }^{4} \mathrm{~T}_{1} \rightarrow$ ${ }^{6} \mathrm{~A}_{1}$. Due to the lack of more detailed information about the intra-ion transitions involving the higher excited states, we will simplify our discussion to the ground state and the first excited state, the latter representing all the excited states of the ion. For $\mathrm{Fe}^{2+}$, the ground and the excited states are ${ }^{5} \mathrm{E}$ and ${ }^{5} \mathrm{~T}_{2}$ with $\mathrm{S}=2 .^{26}$ The relaxation rate for the transition ${ }^{5} \mathrm{~T}_{2} \rightarrow{ }^{5} \mathrm{E}$ in $\mathrm{GaN}$ has not been reported, however, for other III-V materials, such as $\mathrm{InP}, \mathrm{GaAs}$, and $\mathrm{GaP}$, this time is in the $\mu$ s range. 1,27

After the excitation of an electron-hole pair over the $\mathrm{GaN}$ band gap, the electron is captured in the ground state of $\mathrm{Fe}^{3+}$ transforming it into $\mathrm{Fe}^{2+}$. In the case of InP:Fe, it has been shown that such a charge transfer transition creates $\mathrm{Fe}^{2+}$ in its excited state ${ }^{5} \mathrm{~T}_{2} \cdot{ }^{26}$ Considering that properties of $\mathrm{Fe}$ ions in different host materials are similar, ${ }^{1}$ we would expect the same transition to take place in $\mathrm{GaN}$ too. After capturing a hole, $\mathrm{Fe}^{2+}$ transforms back into $\mathrm{Fe}^{3+}$, but not in its ground, but in the excited state. ${ }^{4,5}$ Such transition pattern is characteristic for other III-V:Fe semiconductors as well. ${ }^{28}$ Now, in high pulse repetition rate experiments, the time interval between the pulses is six orders of magnitude smaller than $\mathrm{Fe}^{3+}$ relaxation time; besides, the photoexcited carrier concentration in our experiments is comparable to $[\mathrm{Fe}]$. Consequently, $\mathrm{Fe}^{3+}$ does not have sufficient time to relax to its ground state, making the excited state the prevailing one for the $\mathrm{Fe}^{3+}$ ions. This is the key difference between our experiment and the low carrier density cw photo-Hall or the low pulse repetition rate experiment, in which $\mathrm{Fe}^{3+}$ ions have time to relax to their ground state before arrival of the next optical pulse. It is well known that excited states exhibit higher carrier capture-cross sections. ${ }^{29}$ Thus, this difference of the dominating $\mathrm{Fe}^{3+}$ state is the likely reason for the much larger electron capture coefficient determined from the high pulse repetition rate experiments compared to other studies. The cw photo-Hall and the low pulse frequency measurements of carrier dynamics provide parameters for the electron capture to the ground $\mathrm{Fe}^{3+}$ state. Capture parameters determined in the high pulse repetition rate experiments correspond to electron capture to the excited state of $\mathrm{Fe}^{3+}$. This has important implications for modeling of $\mathrm{GaN}: \mathrm{Fe}$ based optoelectronic devices. For devices aimed at low repetition rate applications, capture cross-sections to the ground $\mathrm{Fe}^{3+}$ state should be used. For the high frequency devices, parameters corresponding to the excited state should be applied.

Now, let us discuss the hole capture to $\mathrm{Fe}^{2+}$. The $\mu$ s long excited state relaxation time is about 2-3 orders of magnitude longer than the time interval between the exciting pulses. Thus, the dominant state of the $\mathrm{Fe}^{2+}$ ions should be the excited state ${ }^{5} \mathrm{~T}_{2}$. Here again, as in the case of $\mathrm{Fe}^{3+}$, we generalize the first excited state as representative for eventual higher excited states that were observed, for instance, in $\mathrm{ZnSe}: \mathrm{Fe} .{ }^{30}$ In the hole energy scale, relevant for the nonradiative hole capture, ${ }^{31}$ the excited state is closer to the valence band than the ground state (Fig. 9) and is a more efficient hole trap than the ground state. ${ }^{31}$ This, along with the Coulomb attraction between a negatively charged $\mathrm{Fe}^{2+}$ ion and a hole in the valence band, could explain the relatively large value of the hole capture coefficient derived from our experiments.

Concerning the $420 \mathrm{~nm}$ PL peak, several experimental observations are noteworthy. First, its energy is similar to the energy interval between the $\mathrm{Fe}^{2+}$ ground state and the valence band. ${ }^{5,24}$ Second, its decay time is close to the hole capture rate evaluated from the band gap time-resolved PL. For example, consider the hole trapping for sample $1.7 \times 10^{18}$. Shortly after the pulse, the $\mathrm{Fe}^{2+}$ concentration is $\sim 1 \times 10^{18} \mathrm{~cm}^{-3}$ (Fig. 7). For the hole capture coefficient of $1.8 \times 10^{-8} \mathrm{~cm}^{3} / \mathrm{s}$, the hole capture time is $56 \mathrm{ps}$, close to the $420 \mathrm{~nm}$ peak PL decay time of $69 \mathrm{ps}$. Third, the $420 \mathrm{~nm}$ peak decay time does not depend on photoexcited hole concentration. This feature is characteristic for transitions between a negatively charged center and a hole bound to it, as discussed for GaP:Fe in Ref. 32. Thus, our experimental results suggest that the $420 \mathrm{~nm}$ peak is caused by the radiative transition between the $\mathrm{Fe}^{2+}$ ground state and a bound hole. Its low intensity can be explained by the weak population of the $\mathrm{Fe}^{2+}$ ground state because of the long $\mathrm{Fe}^{2+}$ relaxation time with respect to the time interval between the exciting pulses.

\section{CONCLUSIONS}

In conclusion, time-resolved PL measurements were performed on heavily $\mathrm{Fe}$ doped $\mathrm{GaN}$ at different temperatures and excitation powers. The peculiar shape of the PL transients clearly demonstrates that the electron capture to $\mathrm{Fe}^{3+}$ centers is faster than the hole capture to $\mathrm{Fe}^{2+}$. Measurements and simulations have allowed determining electron and hole capture coefficients of $5.5 \times 10^{-8} \mathrm{~cm}^{3} / \mathrm{s}$ and $1.8 \times 10^{-8} \mathrm{~cm}^{3} / \mathrm{s}$ at room temperature. Discrepancy between our electron capture coefficient and the literature 
values is assigned to the difference in the $\mathrm{Fe}^{3+}$ states relevant for a particular experimental configuration. In the low carrier density $\mathrm{cw}$ and the low repetition rate pulsed experiments, the electron capture to the ground $\mathrm{Fe}^{3+}$ state plays the main role. For high pulse repetition rates, the excited $\mathrm{Fe}^{3+}$ state is dominant. The temperature dependence of the electron trapping has been found to be weak, showing that potential barrier for the multiphonon electron capture is small. The $420 \mathrm{~nm}$ PL peak is assigned to the radiative transitions between the $\mathrm{Fe}^{2+}$ ground state and a bound hole.

\section{ACKNOWLEDGMENTS}

We would like to thank A. Alkauskas, D. Wickramaratne, and C. G. Van de Walle for fruitful discussions. The work at KTH was performed within the frame of Linnaeus Center for Advanced Optics and Photonics and supported by the Swedish Research Council (Contract No. 621-2013-4096).

${ }^{1}$ E. Malguth, A. Hoffmann, and M. R. Phillips, Phys. Status Solidi B 245, 455 (2008).

${ }^{2}$ T. Aggerstam, A. Pinos, S. Marcinkevičius, M. Linnarsson, and S. Lourdudoss, J. Electron. Mater. 36, 1621 (2007).

${ }^{3}$ T. Dietl, H. Ohno, and F. Matsukura, Phys. Rev. B 63, 195205 (2001)

${ }^{4}$ R. Heitz, P. Maxim, L. Eckey, P. Thurian, A. Hoffmann, I. Broser, K. Pressel, and B. K. Meyer, Phys. Rev. B 55, 4382 (1997).

${ }^{5}$ E. Malguth, A. Hoffmann, W. Gehlhoff, O. Gelhausen, M. R. Phillips, and X. Xu, Phys. Rev. B 74, 165202 (2006)

${ }^{6}$ Y. Fang, X. Wu, J. Yang, Z. Xiao, Y. Yang, F. Zhou, and Y. Song, Appl. Phys. Lett. 107, 051901 (2015).

${ }^{7}$ M. Silvestri, M. J. Uren, and M. Kuball, Appl. Phys. Lett. 102, 073501 (2013).

${ }^{8}$ A. Polyakov, N. Smirnov, A. Govorkov, and S. Pearton, Appl. Phys. Lett. 83, 3314 (2003)

${ }^{9}$ M. A. Reshchikov, Y. T. Moon, X. Gu, B. Nemeth, J. Nause, and H. Morkoç, Physica B 376, 715 (2006).

${ }^{10}$ M. Kubota, T. Onuma, Y. Ishihara, A. Usui, A. Uedono, and S. F. Chichibu, J. Appl. Phys. 105, 083542 (2009).
${ }^{11}$ Z.-Q. Fang, D. C. Look, J. Jasinski, M. Benamara, Z. Liliental-Weber, and R. J. Molnar, Appl. Phys. Lett. 78, 332 (2001).

${ }^{12}$ J. Lyons, A. Janotti, and C. Van de Walle, Appl. Phys. Lett. 97, 152108 (2010).

${ }^{13}$ P. Ščajev, K. Jarašiūnas, S. Okur, Ü. Özgür, and H. Morkoç, J. Appl. Phys. 111, 023702 (2012).

${ }^{14}$ A. Pinos, S. Marcinkevičius, M. Usman, and A. Hallén, Appl. Phys. Lett. 95, 112108 (2009).

${ }^{15}$ D. Söderström, S. Marcinkevičius, S. Karlsson, and S. Lourdudoss, Appl. Phys. Lett. 70, 3374 (1997).

${ }^{16}$ A. Česna, D. Söderström, S. Marcinkevičius, and S. Lourdudoss, J. Appl. Phys. 85, 1234 (1999).

${ }^{17}$ A. Alkauskas, Q. Yan, and C. G. Van de Walle, Phys. Rev. B 90, 075202 (2014).

${ }^{18}$ J. Oila, J. Kivioja, V. Ranki, K. Saarinen, D. C. Look, R. J. Molnar, S. Park, S. Lee, and J. Han, Appl. Phys. Lett. 82, 3433 (2003).

${ }^{19}$ A. Gaarder, S. Marcinkevičius, C. Angulo Barrios, and S. Lourdudoss, Semicond. Sci. Technol. 17, 129 (2002).

${ }^{20}$ R. Aleksiejunas, M. Azize, Z. Bougrioua, T. Malinauskas, S. Nargelas, and K. Jarasiunas, Phys. Status Solidi C 6, S723 (2009).

${ }^{21}$ B. Šantić, Semicond. Sci. Technol. 18, 219 (2003).

${ }^{22}$ J. Shah, Hot Carriers in Semiconductor Nanostructures: Physics and Applications (Elsevier, Philadelphia, 2012).

${ }^{23}$ C. H. Henry and D. V. Lang, Phys. Rev. B 15, 989 (1977).

${ }^{24}$ D. C. Look, J. H. Leach, R. Metzger, T. K. Uždavinys, and S. Marcinkevičius, "Photo-Hall-effect study of excitation and recombination in Fe-doped GaN" (unpublished).

${ }^{25}$ V. F. Agekyan, Phys. Solid State 44, 2013 (2002).

${ }^{26}$ E. Malguth, A. Hoffmann, S. Werner, M. H. Kane, and I. T. Ferguson, in Nitrides and Related Bulk Materials, edited by R. Kniep (Mater. Res. Soc. Symp. Proc., 2008), Vol. 1040, p. Q06.

${ }^{27}$ P. B. Klein, J. E. Furneaux, and R. L. Henry, Phys. Rev. B 29, 1947 (1984).

${ }^{28}$ K. Pressel, G. Bohnert, A. Dörnen, B. Kaufmann, J. Denzel, and K. Thonke, Phys. Rev. B 47, 9411 (1993).

${ }^{29}$ M. Lax, Phys. Rev. 119, 1502 (1960).

${ }^{30}$ J. Peppers, V. V. Fedorov, and S. B. Mirov, Opt. Express 23, 4406 (2015).

${ }^{31}$ K. P. Korona, A. Wysmolek, M. Kamińska, A. Twardowski, M. Piersa, M. Palczewska, G. Strzelecka, A. Hruban, J. Kuhl, R. Adomavicius, and A. Krotkus, Physica B 382, 220 (2006).

${ }^{32}$ P. Roura, T. Benyattou, G. Guillot, R. Moncorge, and W. Ulrici, Phys. Rev. B 45, 11698 (1992). 